\title{
Perturbative ground states of the 1+1d double-well
}

\author{
Hui Liu ${ }^{1,2, a}$, Yao Zhou ${ }^{1,2, b}$, Jarah Evslin ${ }^{1,2, c}$ \\ ${ }^{1}$ Institute of Modern Physics, Chinese Academy of Sciences, Lanzhou 730000, China \\ ${ }^{2}$ University of the Chinese Academy of Sciences, Beijing 100049, China
}

Received: 5 March 2020 / Accepted: 14 April 2020 / Published online: 4 May 2020

(C) The Author(s) 2020

\begin{abstract}
At second order in perturbation theory, we find the ground states of the $\phi^{4}$ double-well quantum field theory in $1+1$ dimensions. The operators which create these ground states from the free vacuum are constructed explicitly at this order, as is the operator which interpolates between the ground states. As a warmup we perform the analogous calculation in quantum mechanics, where the true ground state is unique but in perturbation theory there are also two ground states.
\end{abstract}

\section{Introduction}

One of the greatest triumphs in nonperturbative quantum field theory was the calculation of the leading order correction to the $\phi^{4}$ kink mass in Ref. [1]. The kink mass is the difference between the kink energy and the ground state energy. The kink and vacuum sectors were both regularized by placing them in a periodic box and fixing the number of modes considered, matching the number of modes included in the two sectors. The calculation was so successful that it was performed again by a number of groups using various regulators and matching conditions. In Ref. [2] it was explained that the leading correction has a spurious dependence on the matching of the regulators, with a fixed mode number and sharp energy cutoff leading to distinct results. Since then even more schemes have been proposed, such as demanding that the correction be independent of the topological sector when the mass vanishes [3]. While various consistency checks have been performed, it remains unclear which matching condition, if any, is correct or whether perhaps all are correct but with different definitions of the bare theory or renormalization conditions.

\footnotetext{
a e-mail: liuhui@impcas.ac.cn

be-mail: yaozhou@impcas.ac.cn

c e-mail: jarah@impcas.ac.cn (corresponding author)
}

The fact that the kink mass depends on the regulator is not a problem. The theory itself is defined by choosing a regulator and then a renormalization scheme. Different choices correspond, for example, to different mass terms and so define inequivalent theories, thus one should not expect the corresponding kink masses to agree. The problem above is not that the kink mass depends on the regulator, which is part of the definition of the theory, but rather that it depends on a choice of matching conditions of regulators in different sectors, which is an arbitrary choice and so should not affect physical quantities.

Our approach [4] is different. We regulate and renormalize exactly once, not once for each sector, and so there is no arbitrary choice of matching. States in the topologically nontrivial sector are constructed using a nonlocal operator, which allows their masses to be computed using the original Hamiltonian and circumvents the need to introduce a separate Hamiltonian for soliton sectors. More precisely, we renormalize the theory by normal ordering the vacuum sector alone, and we use the kink operator to show that this already renders all kink sector quantities finite, ${ }^{1}$ with no need to separately regularize or renormalize the kink sector. Almost immediately it had been noted [5] that the regularization of the $\phi^{4}$ theory was not necessary at all, as scalar theories in $2 \mathrm{~d}$ with canonical kinetic terms are manifestly finite when normal-ordered, because the only divergences arise from loops involving a single vertex. Our approach is in this sense manifestly finite.

From the point of view of the original theory, the normal ordering is just another renormalization condition, albeit one whiich removes the need for regularization. We thus suspect that this approach can be generalized to any renormalization condition [6].

Now that no arbitrary matching condition is imposed, all ambiguities have been removed and so one can for the first

\footnotetext{
${ }^{1}$ Normal ordering the vacuum sector does not lead to a normal-ordered kink sector. In fact, the mismatch between these two normal orderings is precisely the one-loop correction to the kink mass [4].
} 
time meaningfully press deeper into the quantum regime. It is now possible to unambiguously calculate the subleading corrections to the kink mass and indeed to the kink state itself. However, to do this, one must first calculate the $\phi^{4}$ vacua and their energies to the same order in perturbation theory. This is the goal of the present paper.

This is a straightforward exercise in perturbation theory. But it has so far escaped attention ${ }^{2}$ for several reasons. First of all, the perturbation theory does not converge [9]. Therefore to describe such models rigorously, one convolves the Hamiltonian with a spatial cutoff [10]. However, were one to include such a cutoff in the double-well theory, it would imply that beyond the cutoff there is a unique minimum of the potential. One might still introduce two states, one with an expectation value of the scalar field near each minimum within the cutoff. However, there would be finite action instantons which mix these states and the true ground state would be a unique, symmetric combination. ${ }^{3}$ Therefore there is no stable kink in this theory.

We will ignore these problems, because our goal is eventually to understand the supersymmetric theory where the divergent vacuum energy at the heart of these problems does not appear. Therefore we will proceed with a perturbative approach with no spatial cutoff.

We begin in Sect. 2 with a description of the corresponding problem in quantum mechanics. Here, just like in the position cutoff quantum field theory, there is a unique ground state [11]. However, at any order in perturbation theory there are in fact two distinct ground states, one localized in each well. We will calculate these ground states and the operators which create them from the harmonic oscillator ground state. Then in Sect. 3 we perform the full computation in quantum field theory, calculating the corrections to the states and energy at second order. We see that the solution is a straightforward generalization of that in quantum mechanics. In Sect. 4 we construct the operator which interpolates between the two ground states. This operator is, in a sense, the $x_{0} \rightarrow \infty$ limit of the operator that creates a kink centered at $x_{0}$.

\section{The double well in quantum mechanics}

The double-well model in quantum mechanics has been solved many times, exactly and approximately. In this section we will review the construction of the ground state(s) in second order perturbation theory, as a warm-up for the very

\footnotetext{
${ }^{2}$ In the case of the single well $\phi^{4}$ theory, operators that create the ground state from the disentangled vacuum have recently been constructed in Refs. $[7,8]$. The theory and renormalization scheme are quite different from ours, for example there is no $\phi^{3}$ potential in the single well, however their quartic term is similar to our corresponding result.

${ }^{3}$ Of course the usual logic is to begin by assuming the existence of a unique vacuum.
}

similar calculation in quantum field theory in Sect. 3. There are several reasons not to solve this model in perturbation theory. One problem is that the perturbation theory does not converge [12]. Of course this is a standard problem in quantum theories, where one is used to having only asymptotic expansions. However in the present case it means that one can expect significant errors in the wave function whenever one is not close to the spatial minimum of the potential about which one defines the expansion.

A more serious problem is that while the Schrodinger equation with no extra degrees of freedom always has a unique ground state solution [11] this is not necessarily true in perturbation theory. In the present case, in perturbation theory there are in fact two ground states, one localized at each minimum of the well. In the full, nonperturbative theory these two states are mixed by instantons with only the symmetric combination being the ground state. However no instantons appear in perturbation theory. This problem is actually an advantage for us, as it mimics the case in quantum field theory, where any such instantons would have infinite action and so there really are two distinct vacua. Recall that in $1+1$ dimensions global symmetries, such as our reflection symmetry, can be spontaneously broken if they are discrete, as noted in comment 3 on page 2 of Ref. [13].

We consider a particle in a double-well potential. The corresponding Hamiltonian is

$H=\frac{p^{2}}{2 m}+k\left(x^{2}-v^{2}\right)^{2}$,

where $m$ is the particle's mass, $k$ and $v$ are positive constants and we have set $\hbar=1$. If the well-separation $2 v$ is sufficient, then in perturbation theory there will be two ground states, centered approximately on $x=v$ and $x=-v$, both with the same energy $E$. We will denote these two states by $|+v\rangle$ and $|-v\rangle$

$H| \pm v\rangle=E| \pm v\rangle$.

Our task is to find $E$ and also the wave functions of the two ground states.

To find the state $|+v\rangle$, we can rewrite the Hamiltonian as an anharmonic oscillator centered on $x=v$

$H=\frac{p^{2}}{2 m}+k\left((x-v)^{4}+4 v(x-v)^{3}+4 v^{2}(x-v)^{2}\right)$.

This can be simplified using the spatial displacement operator

$\mathcal{D}_{\alpha}=\exp (-i \alpha p)$ 
to define a new Hamiltonian $H^{\prime}$ by

$H^{\prime}=\mathcal{D}_{-v} H \mathcal{D}_{-v}^{-1}=\frac{p^{2}}{2 m}+4 k v^{2}\left(x^{2}+\frac{1}{v} x^{3}+\frac{1}{4 v^{2}} x^{4}\right)$,

which again has two ground states. We will be interested in that localized at $x=0$

$$
\begin{aligned}
\left|g_{1}\right\rangle & =\mathcal{D}_{-v}|+v\rangle, \quad H^{\prime}\left|g_{1}\right\rangle=\mathcal{D}_{-v} H \mathcal{D}_{-v}^{-1} \mathcal{D}_{-v}|+v\rangle \\
& =\mathcal{D}_{-v} E|+v\rangle=E\left|g_{1}\right\rangle
\end{aligned}
$$

\subsection{Finding $|+v\rangle$}

To set up our perturbative expansion, let us expand the Hamiltonian about a harmonic oscillator Hamiltonian $H_{0}$

$H^{\prime}=H_{0}+\frac{x_{0}}{v} H_{1}+\left(\frac{x_{0}}{v}\right)^{2} H_{2}$,

where

$$
\begin{aligned}
& H_{0}=\frac{p^{2}}{2 m}+\frac{m \omega^{2} x_{0}^{2}}{2} \frac{x^{2}}{x_{0}^{2}}, \quad H_{1}=\frac{m \omega^{2} x_{0}^{2}}{2} \frac{x^{3}}{x_{0}^{3}}, \\
& H_{2}=\frac{m \omega^{2} x_{0}^{2}}{8} \frac{x^{4}}{x_{0}^{4}}, \quad \omega=\sqrt{\frac{8 k v^{2}}{m}} .
\end{aligned}
$$

Here $x_{0}$ is an arbitrary unit of length, which we introduced to make $x_{0} / v$ dimensionless. We imagine that $x_{0} / v$ is small while $x_{0}, m$ and $\omega$ are fixed, and we will calculate states and energies as a power series in $x_{0} / v$. In this approximation the two wells are well-separated and are treated as two decoupled anharmonic oscillators. Here $H_{0}, H_{1}$ and $H_{2}$ are the leading order, 1 st order and 2 nd order terms in the expansion of the Hamiltonian, and there are no higher order terms.

Let $\left|\psi_{0}\right\rangle$ be the ground state of $H_{0}$

$$
H_{0}\left|\psi_{0}\right\rangle=E_{0}\left|\psi_{0}\right\rangle
$$

We will expand $g_{1}$ as

$$
\left|g_{1}\right\rangle=\left|\psi_{0}\right\rangle+\frac{x_{0}}{v}\left|\psi_{1}\right\rangle+\left(\frac{x_{0}}{v}\right)^{2}\left|\psi_{2}\right\rangle+O\left(\left(\frac{x_{0}}{v}\right)^{3}\right)
$$

and its energy as

$$
E=E_{0}+\frac{x_{0}}{v} E_{1}+\left(\frac{x_{0}}{v}\right)^{2} E_{2}+\mathcal{O}\left(\left(\frac{x_{0}}{v}\right)^{3}\right)
$$

Then to second order in perturbation theory, the Schrodinger equation is

$$
\left(H_{0}+\frac{x_{0}}{v} H_{1}+\left(\frac{x_{0}}{v}\right)^{2} H_{2}\right)\left(\left|\psi_{0}\right\rangle+\frac{x_{0}}{v}\left|\psi_{1}\right\rangle\right.
$$

$$
\begin{aligned}
& \left.+\left(\frac{x_{0}}{v}\right)^{2}\left|\psi_{2}\right\rangle+\mathcal{O}\left(\left(\frac{x_{0}}{v}\right)^{3}\right)\right) \\
= & \left(E_{0}+\frac{x_{0}}{v} E_{1}+\left(\frac{x_{0}}{v}\right)^{2} E_{2}+\mathcal{O}\left(\left(\frac{x_{0}}{v}\right)^{3}\right)\right) \\
& \left(\left|\psi_{0}\right\rangle+\frac{x_{0}}{v}\left|\psi_{1}\right\rangle+\left(\frac{x_{0}}{v}\right)^{2}\left|\psi_{2}\right\rangle+\mathcal{O}\left(\left(\frac{x_{0}}{v}\right)^{3}\right)\right) .
\end{aligned}
$$

To simplify calculations below, we fix unit normalization not on $\left|g_{1}\right\rangle$ but rather on $\left|\psi_{0}\right\rangle$, which fixes the normalization of $\left|g_{1}\right\rangle$ so long as each other term $|n\rangle$ is orthogonal to $\left|\psi_{0}\right\rangle$

$\langle 0 \mid 0\rangle=1, \quad\langle 0 \mid 1\rangle=\langle 0 \mid 2\rangle=\cdots=\langle 0 \mid n\rangle=0$.

Now our task is to express $\left|\psi_{1}\right\rangle$ and $\left|\psi_{2}\right\rangle$ in terms of operators acting on $\left|\psi_{0}\right\rangle$. Let us define the Heisenberg operators $a$ and $a^{\dagger}$ as usual by

$a=\sqrt{\frac{m \omega}{2}}\left(x+\frac{i p}{m \omega}\right), \quad a^{\dagger}=\sqrt{\frac{m \omega}{2}}\left(x-\frac{i p}{m \omega}\right)$,

then (2.8) becomes

$$
\begin{aligned}
& H_{0}=\omega\left(a^{\dagger} a+\frac{1}{2}\right), \quad H_{1}=\sqrt{\frac{\omega}{32 m x_{0}^{2}}}\left(a+a^{\dagger}\right)^{3}, \\
& H_{2}=\frac{1}{32 m x_{0}^{2}}\left(a+a^{\dagger}\right)^{4} .
\end{aligned}
$$

\section{Oth order}

First, the terms of order unity in (2.12) reproduce Eq. (2.9) with the unperturbed energy

$E_{0}=\frac{1}{2} \omega$

1st order

At first order we consider the terms of $O\left(\frac{x_{0}}{v}\right)$ in (2.12),

$H_{0}\left|\psi_{1}\right\rangle+H_{1}\left|\psi_{0}\right\rangle=E_{1}\left|\psi_{0}\right\rangle+E_{0}\left|\psi_{1}\right\rangle$.

In terms of $a$ and $a^{\dagger}$ in (2.15) this is,

$$
\begin{aligned}
& \omega\left(a^{\dagger} a+\frac{1}{2}\right)\left|\psi_{1}\right\rangle+\frac{1}{4 x_{0}} \sqrt{\frac{\omega}{2 m}}\left(a+a^{\dagger}\right)^{3}\left|\psi_{0}\right\rangle \\
& \quad=E_{1}\left|\psi_{0}\right\rangle+E_{0}\left|\psi_{1}\right\rangle .
\end{aligned}
$$

Solving (2.18), subject to the conditions (2.13), we find

$$
E_{1}=0, \quad\left|\psi_{1}\right\rangle=-\frac{1}{12 x_{0} \sqrt{2 m \omega}}\left(9 a^{\dagger}+a^{\dagger} a^{\dagger} a^{\dagger}\right)\left|\psi_{0}\right\rangle .
$$




\section{2nd order}

Finally, we consider the terms of $O\left(\left(\frac{x_{0}}{v}\right)^{2}\right)$ in (2.12),

$H_{0}\left|\psi_{2}\right\rangle+H_{1}\left|\psi_{1}\right\rangle+H_{2}\left|\psi_{0}\right\rangle=E_{0}\left|\psi_{2}\right\rangle+E_{1}\left|\psi_{1}\right\rangle+E_{2}\left|\psi_{0}\right\rangle$.

In terms of $a$ and $a^{\dagger}$,

$$
\begin{aligned}
& \omega\left(a^{\dagger} a+\frac{1}{2}\right)\left|\psi_{2}\right\rangle+\frac{1}{4 x_{0}} \sqrt{\frac{\omega}{2 m}}\left(a+a^{\dagger}\right)^{3}\left|\psi_{1}\right\rangle \\
& +\frac{1}{32 m x_{0}^{2}}\left(a+a^{\dagger}\right)^{4}\left|\psi_{0}\right\rangle=E_{0}\left|\psi_{2}\right\rangle+E_{1}\left|\psi_{1}\right\rangle+E_{2}\left|\psi_{0}\right\rangle .
\end{aligned}
$$

Solving (2.13) and (2.21) we obtain

$$
\begin{aligned}
E_{2} & =-\frac{1}{4 m x_{0}^{2}}, \\
\left|\psi_{2}\right\rangle & =\frac{1}{576 m \omega x_{0}^{2}}\left(189 a^{\dagger^{2}}+27 a^{\dagger^{4}}+a^{\dagger^{6}}\right)\left|\psi_{0}\right\rangle .
\end{aligned}
$$

\section{Putting it all together}

Thus, to the 2 nd order, the energy of the ground state is

$E=E_{0}+\frac{x_{0}}{v} E_{1}+\left(\frac{x_{0}}{v}\right)^{2} E_{2}=\frac{\omega}{2}-\frac{1}{4 m v^{2}}$

and the ground state $\left|g_{1}\right\rangle$ is

$$
\begin{aligned}
\left|g_{1}\right\rangle= & {\left[1-\frac{1}{v} \frac{1}{\sqrt{2 m \omega}}\left(\frac{3}{4} a^{\dagger}+\frac{1}{12} a^{\dagger^{3}}\right)+\frac{1}{v^{2}}\left(\frac{21}{64 m \omega} a^{\dagger^{2}}\right.\right.} \\
& \left.\left.+\frac{3}{64 m \omega} a^{\dagger^{4}}+\frac{1}{576 m \omega} a^{\dagger^{6}}\right)\right]\left|\psi_{0}\right\rangle .
\end{aligned}
$$

We can represent this state by a wave function. Recall that the $n$th excited state of the harmonic oscillator is $\frac{\left(a^{\dagger}\right)^{n}}{\sqrt{n !}}\left|\psi_{0}\right\rangle$, and

$\left\langle x\left|\frac{\left(a^{\dagger}\right)^{n}}{\sqrt{n !}}\right| \psi_{0}\right\rangle=\frac{1}{\sqrt{2^{n} n !}}\left(\frac{m \omega}{\pi}\right)^{1 / 4} e^{-\frac{m \omega x^{2}}{2}} H_{n}(\sqrt{m \omega} x)$,

where $H_{n}$ is the $n$th Hermite polynomial. The wave function of $\left|g_{1}\right\rangle$ is

$$
\begin{aligned}
\phi_{g_{1}}(x)= & \left\langle x \mid g_{1}\right\rangle \\
= & \frac{1}{\pi^{1 / 4}} e^{-\frac{1}{2} m \omega x^{2}}\left[m^{\frac{1}{4}} \omega^{\frac{1}{4}}\right. \\
& -\frac{1}{v}\left(\frac{1}{2} m^{\frac{1}{4}} \omega^{\frac{1}{4}} x+\frac{1}{6} m^{\frac{5}{4}} \omega^{\frac{5}{4}} x^{3}\right) \\
& +\frac{1}{v^{2}}\left(-\frac{41}{192} m^{-\frac{3}{4}} \omega^{-\frac{3}{4}}+\frac{1}{4} m^{\frac{1}{4}} \omega^{\frac{1}{4}} x^{2}\right.
\end{aligned}
$$

$$
\left.\left.+\frac{1}{12} m^{\frac{5}{4}} \omega^{\frac{5}{4}} x^{4}+\frac{1}{72} m^{\frac{9}{4}} \omega^{\frac{9}{4}} x^{6}\right)\right]
$$

which is normalized such that

$\int d x \phi_{g_{1}}^{2}(x)=1+\frac{29}{96 m \omega v^{2}}+\frac{4981}{18432 m^{2} \omega^{2} v^{4}}$.

As a check, one can substitute (2.25) and (2.23) into the full Schrodinger equation,

$$
\begin{aligned}
H^{\prime} \phi_{g_{1}}(x)-E \phi_{g_{1}}(x) & \frac{1}{m \pi^{1 / 4}} e^{-\frac{1}{2} m \omega x^{2}}\left[\frac { 1 } { v ^ { 3 } } \left(-\frac{m^{\frac{1}{4}} \omega^{\frac{1}{4}} x}{8}-\frac{19 m^{\frac{5}{4}} \omega^{\frac{5}{4}} x^{3}}{128}\right.\right. \\
& \left.+\frac{m^{\frac{9}{4}} \omega^{\frac{9}{4}} x^{5}}{16}+\frac{m^{\frac{13}{4}} \omega^{\frac{13}{4}} x^{7}}{48}+\frac{m^{\frac{17}{4}} \omega^{\frac{17}{4}} x^{9}}{144}\right) \\
& \times \frac{1}{v^{4}}\left(-\frac{41 m^{-\frac{3}{4}} \omega^{-\frac{3}{4}}}{768}+\frac{m^{\frac{1}{4}} \omega^{\frac{1}{4}} x^{2}}{16}-\frac{3 m^{\frac{5}{4}} \omega^{\frac{5}{4}} x^{4}}{512}\right. \\
& \left.\left.+\frac{5 m^{\frac{9}{4}} \omega^{\frac{9}{4}} x^{6}}{144}+\frac{m^{\frac{13}{4}} \omega^{\frac{13}{4}} x^{8}}{96}+\frac{m^{\frac{17}{4}} \omega^{\frac{17}{4}} x^{10}}{576}\right)\right] \\
= & \mathcal{O}\left(\left(\frac{1}{v}\right)^{3}\right) .
\end{aligned}
$$

As expected, the three leading terms have all canceled, leaving only higher order terms. Of course these higher order terms are large when $x$ is far from the bottom of the well centered at $x=0$, as they will be at any order in perturbation theory.

One may obtain the ground state wave function $|+v\rangle$ of the original Hamiltonian $H$ using the shift operator

$$
\begin{aligned}
\phi_{+v}(x)= & \mathcal{D}_{+v} \phi_{g_{1}}(x) \\
= & \frac{1}{\pi^{1 / 4}} e^{-\frac{1}{2} m \omega(x-v)^{2}}\left[m^{\frac{1}{4}} \omega^{\frac{1}{4}}-\frac{1}{v}\right. \\
& \times\left(\frac{1}{2} m^{\frac{1}{4}} \omega^{\frac{1}{4}}(x-v)+\frac{1}{6} m^{\frac{5}{4}} \omega^{\frac{5}{4}}(x-v)^{3}\right) \\
& +\frac{1}{v^{2}}\left(-\frac{41}{192} m^{-\frac{3}{4}} \omega^{-\frac{3}{4}}+\frac{1}{4} m^{\frac{1}{4}} \omega^{\frac{1}{4}}(x-v)^{2}\right. \\
& \left.\left.+\frac{1}{12} m^{\frac{5}{4}} \omega^{\frac{5}{4}}(x-v)^{4}+\frac{1}{72} m^{\frac{9}{4}} \omega^{\frac{9}{4}}(x-v)^{6}\right)\right] .
\end{aligned}
$$

We believe that it is a perturbative solution in the following sense. Fix some positive $\epsilon \ll v$. It is straightforward to carry out the same calculation as in Sect. 2 to any order $n$ in $1 / v$ and then apply the displacement operator to obtain the order $n$ version of (2.28). We claim that in the interval $[v-\epsilon, v+\epsilon]$ this series will approach the true wave function in the sense of an asymptotic expansion in $\epsilon / v$. This can be seen from the expansion in (2.5). If $x \rightarrow x-v$, orders will be mixed. However this interval corresponds to $|x|<\epsilon$ in which case 
the 3 terms in the right part of the equation have different orders of $\epsilon / v$. This restriction to an interval may seem rather drastic, but in QFT it corresponds to a restriction on the field values and corresponds to the usual intuition that nonperturbative physics is essential for understanding excursions of the fields from one basin of the potential to another.

Note that this shift mixes the orders in our perturbative expansion, and so this wave function is not a solution to the original Schrodinger equation beyond the leading order. In fact, we have not defined a perturbative expansion of the original Hamiltonian $H$.

To obtain the corresponding quantities for the state $|-v\rangle$, one need only change the sign of $v$ in the formulas above.

\section{Constructing a perturbative $\phi^{4}$ ground state}

We are interested in the $1+1$ dimensional quantum field theory of a real scalar field $\phi$ with a double-well $\phi^{4}$ interaction

$H=\int d x: \frac{1}{2}\left(\partial_{x} \phi\right)^{2}+\frac{1}{2} \pi^{2}+\frac{\lambda}{4}\left(\phi^{2}-v^{2}\right)^{2}:$

where the normal ordering is defined below and $\pi$ is the momentum conjugate to $\phi$. We will also define a mass

$m=v \sqrt{2 \lambda}$

which corresponds to the mass of the field when expanded about the bottom of either well. In $1+1$ dimensions $\phi$ is dimensionless, $\lambda$ has dimensions of $m^{2}$ and $v$ is dimensionless. We will consider a perturbative expansion in $1 / v$. This theory has two degenerate ground states of energy $E$, centered near $\phi=v$ and $\phi=-v$, which we will call $|+v\rangle$ and $|-v\rangle$ respectively. In this section we will construct $|+v\rangle$.

Although $\phi$ is not a free field, in the Schrodinger picture we can Fourier transform $\phi$ and $\pi$ to define $a$ and $a^{\dagger}$

$\phi(x)=\int \frac{d p}{2 \pi} \frac{1}{\sqrt{2 \omega_{p}}}\left(a_{p}+a_{-p}^{\dagger}\right) e^{i p x}$,

$\pi(x)=\int \frac{d p}{2 \pi}(-i) \sqrt{\frac{\omega_{p}}{2}}\left(a_{p}-a_{-p}^{\dagger}\right) e^{i p x}$

where

$\omega_{p}=\sqrt{m^{2}+p^{2}}$.

Now the canonical commutation relations

$[\phi(x), \pi(y)]=i \delta(x-y)$

imply that $a$ and $a^{\dagger}$ obey the Heisenberg algebra

$\left[a_{p}, a_{q}^{\dagger}\right]=2 \pi \delta(p-q)$ even in the interacting theory. Our normal ordering prescription is that all $a^{\dagger}$ be placed to the left of all $a$. Note that we normal order the Schrodinger picture field, which, as described in Ref. [14] is useful in this context and is distinct from the more comment interaction picture normal ordering.

There are two distinct interpretations of our normal ordering. One is that the normal-ordered Hamiltonian defines a finite theory which is interesting in its own right. It has a nonvanishing vacuum energy density which we calculate at leading order and also it has a kink sector whose ground state received a one-loop correction which we have calculated in Ref. [15].

Another interpretation is that the Hamiltonian without normal ordering is the fundamental Hamiltonian, and the normal-ordering is a choice of renormalization condition in which one adds an infinite counterterm proportional to $\left[a, a^{\dagger}\right]$. This renormalized theory is finite and so no regularization is necessary. With a different renormalization condition, a regulator would have been necessary and we think that our approach could be applied to that case as well although we have not yet tried. With this second interpretation, one may ask whether we have introduced any ambiguity by choosing a renormalization condition?

In this case the original theory is infinite, and so to make sense of it one needs to make a choice. This choice of renormalization condition and regulator is very physical, it is part of the definition of the theory, and one expects observables to depend on it. It is quite a separate issue from the ambiguity which motivates our work [16] which is a dependence of the kink mass on a choice of matching of two regulators, one for the kink sector and one for the vacuum sector. Masses of states are eigenvalues of the Hamiltonian. Therefore once the Hamiltonian is renormalized/regularized, the masses of all states are already well-defined, and so cannot depend on any auxilliary choice of matching conditions. In Ref. [15] we introduce a unitary shift operator which maps one sector to the other. This operator can be used to express any desired quantity in terms of vacuum sector quantities, and so only the vacuum sector need be regularized. Therefore there is no longer any need to introduce two regulators and match them.

\subsection{The displacement operator $\mathcal{D}$}

The displacement operator

$\mathcal{D}_{\alpha}=e^{-i \alpha \int d x \pi(x)}=e^{\alpha \sqrt{m / 2}\left(a_{0}^{\dagger}-a_{0}\right)}$

shifts the value of $\phi$ by $\alpha$

$\left[\phi, \mathcal{D}_{\alpha}\right]=\alpha \mathcal{D}_{\alpha}$ 
Also it is compatible with normal ordering in the sense that it satisfies the identity

$: F(\pi(x), \phi(x)): \mathcal{D}_{f}=\mathcal{D}_{f}: F(\pi(x), \phi(x)+f(x)):$

for any function $F$.

Applying it to the Hamiltonian $H$ one finds the shifted Hamiltonian

$H^{\prime}=\mathcal{D}_{-v} H \mathcal{D}_{-v}^{-1}, \quad \mathcal{D}_{-v}^{-1}=\mathcal{D}_{+v}$

given by

$$
\begin{aligned}
H^{\prime}= & \int d x: \frac{1}{2}\left(\partial_{x} \phi\right)^{2}+\frac{1}{2} \pi^{2} \\
& +\frac{1}{2} m^{2} \phi^{2}+\frac{1}{v} \frac{m^{2}}{2} \phi^{3}+\frac{1}{v^{2}} \frac{m^{2}}{8} \phi^{4}: .
\end{aligned}
$$

\subsection{Finding the operator perturbatively}

We denote a ground state of (3.11) by $\left|g_{1}\right\rangle$. As $H$ and $H^{\prime}$ are related by the similarity transformation (3.10) they have the same spectra and so the same minimal eigenvalue $E$

$H^{\prime}\left|g_{1}\right\rangle=E\left|g_{1}\right\rangle, \quad H| \pm v\rangle=E| \pm v\rangle$.

We claim that

$|+v\rangle=\mathcal{D}_{-v}^{-1}\left|g_{1}\right\rangle$

Note that this is consistent with (3.12) as

$H \mathcal{D}_{-v}^{-1}\left|g_{1}\right\rangle=\mathcal{D}_{-v}^{-1} H^{\prime}\left|g_{1}\right\rangle=E \mathcal{D}_{-v}^{-1}\left|g_{1}\right\rangle$

establishing that $\mathcal{D}_{-v}^{-1}\left|g_{1}\right\rangle$ is indeed a minimal energy eigenstate of $H$. Equation (2.6) thus applies as written to the quantum field theory case. Again our goal is to write $\left|g_{1}\right\rangle$ in terms of the free ground state.

First we perturbatively expand the Hamiltonian (3.11),

$H^{\prime}=H_{0}+\frac{1}{v} H_{1}+\frac{1}{v^{2}} H_{2}$

where

$$
\begin{aligned}
& H_{0}=\int d x: \frac{1}{2} \phi^{\prime 2}+\frac{1}{2} \pi^{2}+\frac{1}{2} m^{2} \phi^{2}: \\
& H_{1}=\frac{1}{v} \int d x \frac{m^{2}}{2}: \phi^{3}:, \quad H_{2}=\frac{1}{v^{2}} \int d x \frac{m^{2}}{8}: \phi^{4}:
\end{aligned}
$$

$H_{0}$ is the Hamiltonian of the free, massive Klein-Gordon theory, and the next two terms are perturbations of order $O\left(\frac{1}{v}\right)$ and $O\left(\frac{1}{v^{2}}\right)$.
Expanding the ground state

$\left|g_{1}\right\rangle=\left(\left|\psi_{0}\right\rangle+\frac{1}{v}\left|\psi_{1}\right\rangle+\frac{1}{v^{2}}\left|\psi_{2}\right\rangle\right)+\mathcal{O}\left(\frac{1}{v^{3}}\right)$

subject to (2.13) and the energy as in Eq. (2.11) we obtain the eigenvalue equation for $\left|g_{1}\right\rangle$

$$
\begin{aligned}
& \left(H_{0}+\frac{1}{v} H_{1}+\frac{1}{v^{2}} H_{2}\right) \\
& \left(\left|\psi_{0}\right\rangle+\frac{1}{v}\left|\psi_{1}\right\rangle+\frac{1}{v^{2}}\left|\psi_{2}\right\rangle+\mathcal{O}\left(\frac{1}{v^{3}}\right)\right) \\
& =\left(E_{0}+\frac{1}{v} E_{1}+\frac{1}{v^{2}} E_{2}+\mathcal{O}\left(\frac{1}{v^{3}}\right)\right) \\
& \quad\left(\left|\psi_{0}\right\rangle+\frac{1}{v}\left|\psi_{1}\right\rangle+\frac{1}{v^{2}}\left|\psi_{2}\right\rangle+\mathcal{O}\left(\frac{1}{v^{3}}\right)\right) .
\end{aligned}
$$

Oth order

The leading terms in (3.18) are

$H_{0}\left|\psi_{0}\right\rangle=E_{0}\left|\psi_{0}\right\rangle$

so that $\left|\psi_{0}\right\rangle$ is the free ground state and

$E_{0}=0$.

Note that this is superficially different from the energy in the quantum mechanical case, because we have normal ordered our Hamiltonian. Of course had we normal ordered in that case we would also have obtained a vanishing value of $E_{0}$.

\section{1st order}

The first order terms in (3.18) are

$H_{0}\left|\psi_{1}\right\rangle+H_{1}\left|\psi_{0}\right\rangle=E_{1}\left|\psi_{0}\right\rangle$

Using the normal ordering in $H_{1}$ and the fact that

$a_{p}\left|\psi_{0}\right\rangle=0$

as a result of (3.19) and (3.20), this can be written explicitly as

$H_{0}\left|\psi_{1}\right\rangle=E_{1}\left|\psi_{0}\right\rangle-\frac{m^{2}}{2} \int \frac{d p_{1} d p_{2}}{(2 \pi)^{2}} \frac{a_{p_{1}}^{\dagger} a_{p_{2}}^{\dagger} a_{-p_{1}-p_{2}}^{\dagger}}{\sqrt{8 \omega_{p_{1}} \omega_{p_{2}} \omega_{p_{1}+p_{2}}}}\left|\psi_{0}\right\rangle$.

Applying $\langle 0|$ to (3.23), together with the condition (2.13), we find

$E_{1}=0$ 
and so then (3.23) is solved by inverting $H_{0}$, yielding a sum over frequencies in the denominator

$$
\begin{aligned}
\left|\psi_{1}\right\rangle= & -\frac{m^{2}}{2} \int \frac{d p_{1} d p_{2}}{(2 \pi)^{2}} \frac{1}{\sqrt{8 \omega_{p_{1}} \omega_{p_{2}} \omega_{p_{1}+p_{2}}}} \\
& \times \frac{a_{p_{1}}^{\dagger} a_{p_{2}}^{\dagger} a_{-p_{1}-p_{2}}^{\dagger}}{\left(\omega_{p_{1}}+\omega_{p_{2}}+\omega_{p_{1}+p_{2}}\right)}\left|\psi_{0}\right\rangle .
\end{aligned}
$$

This looks similar to (2.19), except that the $a^{\dagger}$ term is missing. This again is the result of the normal ordering.

\section{2nd order}

Finally we arrive at the $O\left(\frac{1}{v^{2}}\right)$ terms in (3.18),

$H_{0}\left|\psi_{2}\right\rangle+H_{1}\left|\psi_{1}\right\rangle+H_{2}\left|\psi_{0}\right\rangle=E_{2}\left|\psi_{0}\right\rangle$.

Applying $\langle 0|$ on the left, the first term vanishes because $E_{0}=$ 0 and the $H_{2}$ term vanishes because it is normal ordered. Using the normalization of $|0\rangle$ we obtain

$$
E_{2}=\left\langle 0\left|H_{1}\right| \psi_{1}\right\rangle
$$

Using (3.25) we find the ground state energy at this order

$$
\begin{aligned}
& E_{2}= \int d x \mathcal{E}_{2}(x) \\
& \mathcal{E}_{2}(x)=-\frac{3}{16} m^{4} \int \frac{d p_{1}}{2 \pi} \frac{d p_{2}}{2 \pi} \frac{1}{\omega_{p_{1}} \omega_{p_{2}} \omega_{p_{1}+p_{2}}} \\
& \times \frac{1}{\left(\omega_{p_{1}}+\omega_{p_{2}}+\omega_{p_{1}+p_{2}}\right)} .
\end{aligned}
$$

The energy is infinite, however the energy density $\mathcal{E}_{2}$ is finite and constant. To the 2 nd order, the energy density of the $\phi^{4}$ vacuum state is

$$
\begin{aligned}
\mathcal{E}= & 0+\frac{1}{v} \mathcal{E}_{1}+\frac{1}{v^{2}} \mathcal{E}_{2} \\
= & -\frac{1}{v^{2}} \frac{3}{16} m^{4} \int \frac{d p_{1}}{2 \pi} \frac{d p_{2}}{2 \pi} \frac{1}{\omega_{p_{1}} \omega_{p_{2}} \omega_{p_{1}+p_{2}}} \\
& \times \frac{1}{\left(\omega_{p_{1}}+\omega_{p_{2}}+\omega_{p_{1}+p_{2}}\right)} \\
\approx & -0.022 \lambda .
\end{aligned}
$$

Now we know everything in Eq. (3.26) except for $\left|\psi_{2}\right\rangle$. This can again be found by inverting $H_{0}$, providing another sum over frequencies in the denominator

$$
\begin{aligned}
& \left|\psi_{2}\right\rangle=\left[\frac{9}{4} m^{4} \int \frac{\prod_{i=1}^{2} d p_{i}}{(2 \pi)^{2}}\right.
\end{aligned}
$$

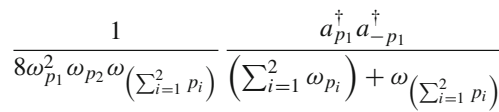

$$
\begin{aligned}
& +\int \frac{\prod_{i=1}^{3} d p_{i}}{(2 \pi)^{3}} \frac{1}{\sqrt{16\left(\prod_{i=1}^{3} \omega_{p_{i}}\right) \omega\left(\sum_{i=1}^{3} p_{i}\right)}}
\end{aligned}
$$

$$
\begin{aligned}
& \times \frac{\left(\prod_{i=1}^{3} a_{p_{i}}^{\dagger}\right) a_{\left(-\sum_{i=1}^{3} p_{i}\right)}^{\dagger}}{\left(\sum_{i=1}^{3} \omega_{p_{i}}\right)+\omega\left(\sum_{i=1}^{3} p_{i}\right)} \\
& \times\left(\frac{9}{4} m^{4} \frac{1}{2 \omega\left(\sum_{i=1}^{2} p_{i}\right)\left(\omega_{\left(\sum_{i=1}^{2} p_{i}\right)}+\omega_{p_{3}}+\omega_{\left(\sum_{i=1}^{3} p_{i}\right)}\right)}-\frac{m^{2}}{8}\right) \\
& +\int \frac{\prod_{i=1}^{4} d p_{i}}{(2 \pi)^{4}} \\
& \frac{m^{4}}{4} \frac{1}{\left(\sum_{i=1}^{4} \omega_{p_{i}}\right)+\omega_{\left(\sum_{i=1}^{2} p_{i}\right)}+\omega_{\left(\sum_{i=3}^{4} p_{i}\right)}} \\
& \times \frac{1}{\sqrt{64\left(\prod_{i=1}^{4} \omega_{p_{i}}\right) \omega\left(\sum_{i=1}^{2} p_{i}\right)^{\omega}\left(\sum_{i=3}^{4} p_{i}\right)}} \\
& \left.\times \frac{\left(\prod_{i=1}^{4} a_{p_{i}}^{\dagger}\right) a_{\left(-\sum_{i=1}^{\dagger} p_{i}\right)}^{a^{\dagger}\left(-\sum_{i=3}^{4} p_{i}\right)}}{\left(\sum_{i=3}^{4} \omega_{p_{i}}\right)+\omega\left(\sum_{i=3}^{4} p_{i}\right)}\right]\left|\psi_{0}\right\rangle .
\end{aligned}
$$

Note that it resembles the corresponding expression in quantum mechanics (2.22). The two particle, four particle and six particle components have just the same origin in both cases.

Now we can write $\left|g_{1}\right\rangle$ to 2 nd order

$$
\begin{aligned}
& \left|g_{1}\right\rangle=\left\{1-\frac{1}{v} \frac{m^{2}}{2} \int \frac{d p_{1} d p_{2}}{(2 \pi)^{2}} \frac{1}{\sqrt{8 \omega_{p_{1}} \omega_{p_{2}} \omega_{p_{1}+p_{2}}}}\right. \\
& \times \frac{a_{p_{1}}^{\dagger} a_{p_{2}}^{\dagger} a_{-p_{1}-p_{2}}^{\dagger}}{\left(\omega_{p_{1}}+\omega_{p_{2}}+\omega_{p_{1}+p_{2}}\right)} \\
& +\frac{1}{v^{2}}\left[\frac{9}{4} m^{4} \int \frac{d p_{1} d p_{2}}{(2 \pi)^{2}} \frac{1}{8 \omega_{p_{1}}^{2} \omega_{p_{2}} \omega_{p_{1}+p_{2}}}\right. \\
& \times \frac{a_{p_{1}}^{\dagger} a_{-p_{1}}^{\dagger}}{\omega_{p_{1}}+\omega_{p_{2}}+\omega_{p_{1}+p_{2}}} \\
& +\int \frac{\prod_{i=1}^{3} d p_{i}}{(2 \pi)^{3}} \frac{1}{\sqrt{16\left(\prod_{i=1}^{3} \omega_{p_{i}}\right) \omega\left(\sum_{i=1}^{3} p_{i}\right)}} \\
& \times \frac{\left(\prod_{i=1}^{3} a_{p_{i}}^{\dagger}\right) a_{-\sum_{i=1}^{\dagger} p_{i}}^{\dagger}}{\left(\sum_{i=1}^{3} \omega_{p_{i}}\right)+\omega_{\sum_{i=1}^{3} p_{i}}} \\
& \times\left(\frac{9}{4} m^{4} \frac{1}{2 \omega_{p_{1}+p_{2}}\left(\omega_{p_{1}+p_{2}}+\omega_{p_{3}}+\omega_{\sum_{i=1}^{3} p_{i}}\right)}-\frac{m^{2}}{8}\right) \\
& +\int \frac{\prod_{i=1}^{4} d p_{i}}{(2 \pi)^{4}} \frac{m^{4}}{4} \frac{1}{\left(\sum_{i=1}^{4} \omega_{p_{i}}\right)+\omega_{p_{1}+p_{2}}+\omega_{p_{3}+p_{4}}} \\
& \times \frac{1}{\sqrt{64\left(\prod_{i=1}^{4} \omega_{p_{i}}\right) \omega_{p_{1}+p_{2}} \omega_{p_{3}+p_{4}}}}
\end{aligned}
$$

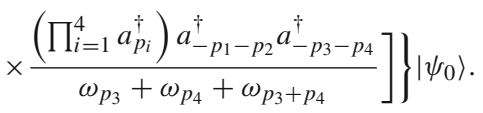

Let us denote the operator in the curly bracket by $\mathcal{O}^{\prime}$

$\left|g_{1}\right\rangle=\mathcal{O}^{\prime}\left|\psi_{0}\right\rangle$ 
Let the operator which takes the free vacuum $\left|\psi_{0}\right\rangle$ to the $\phi^{4}$ vacuum $|+v\rangle$ be $\mathcal{O}_{1}$

$$
|+v\rangle=\mathcal{O}_{1}\left|\psi_{0}\right\rangle
$$

then

$\mathcal{O}_{1}=\mathcal{D}_{+v} \mathcal{O}^{\prime}$

\section{Interpolating operator}

To obtain $|-v\rangle$, the other ground state, we perform exactly the same manipulations but with the sign of $v$ flipped. The resulting state $\left|g_{1}\right\rangle$ is identical to that in Eq. (3.32) except each $v$ is replaced by $-v$. Let the corresponding operator, corresponding to $\mathcal{O}^{\prime}$ with $v \rightarrow-v$, be $\mathcal{O}^{\prime \prime}$ and the operator that changes $\left|\psi_{0}\right\rangle$ to $|-v\rangle$ be $\mathcal{O}_{2}$

$\mathcal{O}_{2}=\mathcal{D}_{-v} \mathcal{O}^{\prime \prime}$

The operator $\mathcal{O}$ that changes $|+v\rangle$ to $|-v\rangle$

$|-v\rangle=\mathcal{O}|+v\rangle$

is then

$$
\begin{aligned}
\mathcal{O} & =\mathcal{O}_{2} \mathcal{O}_{1}^{-1} \\
& =\mathcal{D}_{-v} \mathcal{O}^{\prime \prime}\left(\mathcal{D}_{+v} \mathcal{O}^{\prime}\right)^{-1} \\
& =\mathcal{D}_{-v} \mathcal{O}^{\prime \prime} \mathcal{O}^{-1} \mathcal{D}_{-v}
\end{aligned}
$$

Let us define the shorthand notation

$$
\begin{aligned}
\mathcal{O}^{\prime} & =1-\frac{1}{v} \mathcal{A}+\frac{1}{v^{2}} \mathcal{B}, \\
\mathcal{O}^{\prime \prime} & =1+\frac{1}{v} \mathcal{A}+\frac{1}{v^{2}} \mathcal{B}
\end{aligned}
$$

where

$$
\begin{aligned}
& \mathcal{A}=\frac{m^{2}}{2} \int \frac{d p_{1} d p_{2}}{(2 \pi)^{2}} \frac{1}{\sqrt{8 \omega_{p_{1}} \omega_{p_{2}} \omega_{p_{1}+p_{2}}}} \\
& \times \frac{a_{p_{1}}^{\dagger} a_{p_{2}}^{\dagger} a_{-p_{1}-p_{2}}^{\dagger}}{\left(\omega_{p_{1}}+\omega_{p_{2}}+\omega_{p_{1}+p_{2}}\right)} \\
& \mathcal{B}=\frac{9}{4} m^{4} \int \frac{\prod_{i=1}^{2} d p_{i}}{(2 \pi)^{2}} \frac{1}{8 \omega_{p_{1}}^{2} \omega_{p_{2}} \omega}\left(\sum_{i=1}^{2} p_{i}\right) \\
& \times \frac{a_{p_{1}}^{\dagger} a_{-p_{1}}^{\dagger}}{\left(\sum_{i=1}^{2} \omega_{p_{i}}\right)+\omega_{\left(\sum_{i=1}^{2} p_{i}\right)}} \\
& +\int \frac{\prod_{i=1}^{3} d p_{i}}{(2 \pi)^{3}} \frac{1}{\sqrt{16\left(\prod_{i=1}^{3} \omega_{p_{i}}\right) \omega\left(\sum_{i=1}^{3} p_{i}\right)}} \\
& \times \frac{\left(\prod_{i=1}^{3} a_{p_{i}}^{\dagger}\right) a_{\left(-\sum_{i=1}^{\dagger} p_{i}\right)}^{\dagger}}{\left(\sum_{i=1}^{3} \omega_{p_{i}}\right)+\omega_{\left(\sum_{i=1}^{3} p_{i}\right)}}
\end{aligned}
$$

$$
\begin{aligned}
& \times\left(\frac{9}{4} m^{4} \frac{1}{2 \omega_{\left(\sum_{i=1}^{2} p_{i}\right)}\left(\omega_{\left(\sum_{i=1}^{2} p_{i}\right)}+\omega_{p_{3}}+\omega_{\left(\sum_{i=1}^{3} p_{i}\right)}\right)}-\frac{m^{2}}{8}\right) \\
& +\int \frac{\prod_{i=1}^{4} d p_{i}}{(2 \pi)^{4}} \frac{m^{4}}{4} \frac{1}{\left(\sum_{i=1}^{4} \omega_{p_{i}}\right)+\omega\left(\sum_{i=1}^{2} p_{i}\right)+\omega\left(\sum_{i=3}^{4} p_{i}\right)}
\end{aligned}
$$

$$
\begin{aligned}
& \times \frac{1}{\sqrt{64\left(\prod_{i=1}^{4} \omega_{p_{i}}\right) \omega\left(\sum_{i=1}^{2} p_{i}\right)^{\omega}\left(\sum_{i=3}^{4} p_{i}\right)}} \\
& \times \frac{\left(\prod_{i=1}^{4} a_{p_{i}}^{\dagger} a_{\left(-\sum_{i=1}^{\dagger} p_{i}\right)^{\dagger}\left(-\sum_{i=3}^{4} p_{i}\right)}^{\left(\sum_{i=3}^{4} \omega_{p_{i}}\right)+\omega\left(\sum_{i=3}^{4} p_{i}\right)}\right.}{}
\end{aligned}
$$

Then,

$$
\begin{aligned}
\mathcal{O}^{\prime-1} & =\left(1-\frac{1}{v} \mathcal{A}+\frac{1}{v^{2}} \mathcal{B}\right)^{-1} \\
& =1+\frac{1}{v} \mathcal{A}+\frac{1}{v^{2}}\left(\mathcal{A}^{2}-\mathcal{B}\right) .
\end{aligned}
$$

Finally,

$$
\begin{aligned}
\mathcal{O}= & \mathcal{D}_{-v}\left(1+\frac{1}{v} \mathcal{A}+\frac{1}{v^{2}} \mathcal{B}\right)\left(1+\frac{1}{v} \mathcal{A}+\frac{1}{v^{2}}\left(\mathcal{A}^{2}-\mathcal{B}\right)\right) \mathcal{D}_{-v} \\
= & \mathcal{D}_{-v}\left[1+\frac{2}{v} \mathcal{A}+\frac{2}{v^{2}} \mathcal{A}^{2}\right] \mathcal{D}_{-v} \\
= & e^{-v \sqrt{\frac{m}{2}}\left(a_{0}^{\dagger}-a_{0}\right)}\left[1+\frac{m^{2}}{v} \int \frac{d p_{1} d p_{2}}{(2 \pi)^{2}} \frac{1}{\sqrt{8 \omega_{p_{1}} \omega_{p_{2}} \omega_{p_{1}+p_{2}}}}\right. \\
& \times \frac{a_{p_{1}}^{\dagger} a_{p_{2}}^{\dagger} a_{-p_{1}-p_{2}}^{\dagger}}{\left(\omega_{p_{1}}+\omega_{p_{2}}+\omega_{p_{1}+p_{2}}\right)} \\
& +\frac{m^{4}}{2 v^{2}}\left(\int \frac{d p_{1} d p_{2}}{(2 \pi)^{2}} \frac{1}{\sqrt{8 \omega_{p_{1}} \omega_{p_{2}} \omega_{p_{1}+p_{2}}}}\right. \\
& \left.\left.\times \frac{a_{p_{1}}^{\dagger} a_{p_{2}}^{\dagger} a_{-p_{1}-p_{2}}^{\dagger}}{\left(\omega_{p_{1}}+\omega_{p_{2}}+\omega_{p_{1}+p_{2}}\right)}\right)^{2}\right] e^{-v \sqrt{\frac{m}{2}}\left(a_{0}^{\dagger}-a_{0}\right)} .
\end{aligned}
$$

At this order, there is no dependence on $\mathcal{B}$.

The main motivation of the present work is to provide necessary formulas for the calculation of higher corrections to the $\phi^{4}$ kink. If the center of the kink is at $x_{0}$ and the kink form factor, in the sense of Ref. [17], interpolates between $-v$ at $x \rightarrow-\infty$ and $+v$ at $x \rightarrow \infty$, then we expect that in the suitably defined limit $x_{0} \rightarrow \infty$ the operator which creates the kink will reduce to $\mathcal{O}$.

\section{Remarks}

In this note we have performed a simple exercise in perturbative quantum field theory, finding the ground states of the $\phi^{4}$ double-well theory and their energies. Armed with this perturbative expression for the ground state, we may now extend the calculation of kink state and mass in Ref. [4] beyond the leading quantum corrections. We wish to do this because our eventual goal, in the supersymmetric case, is to attempt to construct the kink at all orders but we would like the first terms in the perturbation theory as a starting point. 
Another possible application of this ground state is in Higgs physics. The Higgs field is of this form, except that it is complex and also lives in $3+1$ dimensions. However the radial mode, near the minimum, is governed by the same potential. A quantum description of this state may help one to study the following phenomena.

A superconductor can repel a magnetic field. It does this by letting its electrons travel in circles, which creates a counter magnetic field. This costs energy, and so changes the potential energy as a function of the Cooper pair condensate. The field equations imply that this energy must be minimized and so, in the presence of an external magnetic field, it is well known that the Cooper pair condensate is reduced.

Exactly the same argument may be applied to the Standard Model Higgs field. It creates mass for, for example, the top quark. But this mass costs the Higgs field energy, and so in the minimum energy field configuration, in the presence of a top quark, the Higgs condensate will be reduced. This reduction is quite localized about the top quark, which anyway does not live long. However, in a proposed $100 \mathrm{TeV}$ collider, one expects that there will be a larger region where a number of particles are given masses by the Higgs field. If this energy density is of order the Higgs potential, then the backreaction on the Higgs field, reducing its value and the particle masses, may be considerable. This would be new physics, but simply calculated in perturbation theory with this potential coupled via a Yukawa coupling to fermions. This is the same coupling that we will need to add anyway to study the supersymmetric kink.

Acknowledgements JE is supported by the CAS Key Research Program of Frontier Sciences grant QYZDY-SSW-SLH006 and the NSFC MianShang grants 11875296 and 11675223 . JE also thanks the Recruitment Program of High-end Foreign Experts for support.

Data Availability Statement This manuscript has no associated data or the data will not be deposited. [Authors' comment: This paper reports an analytic calculation. There is no data because there was no experiment and, while we did perform some numerical calculations, they were consistent with the analytical calculations and so not of sufficient interest to include in our publication.]

Open Access This article is licensed under a Creative Commons Attribution 4.0 International License, which permits use, sharing, adaptation, distribution and reproduction in any medium or format, as long as you give appropriate credit to the original author(s) and the source, provide a link to the Creative Commons licence, and indicate if changes were made. The images or other third party material in this article are included in the article's Creative Commons licence, unless indicated otherwise in a credit line to the material. If material is not included in the article's Creative Commons licence and your intended use is not permitted by statutory regulation or exceeds the permitted use, you will need to obtain permission directly from the copyright holder. To view a copy of this licence, visit http://creativecomm ons.org/licenses/by/4.0/.

Funded by SCOAP ${ }^{3}$.

\section{References}

1. R.F. Dashen, B. Hasslacher, A. Neveu, Nonperturbative methods and extended hadron models in field theory 2. Two-dimensional models and extended hadrons. Phys. Rev. D 10, 4130 (1974). https://doi.org/10.1103/PhysRevD.10.4130

2. A. Rebhan, P. van Nieuwenhuizen, No saturation of the quantum Bogomolnyi bound by two-dimensional supersymmetric solitons. Nucl. Phys. B 508, 449 (1997). https://doi.org/10.1016/ S0550-3213(97)00625-1. arXiv:hep-th/9707163

3. H. Nastase, M.A. Stephanov, P. van Nieuwenhuizen, A. Rebhan, Topological boundary conditions, the BPS bound, and elimination of ambiguities in the quantum mass of solitons. Nucl. Phys. B 542, 471 (1999). https://doi.org/10.1016/ S0550-3213(98)00773-1. arXiv:hep-th/9802074

4. J. Evslin, Manifestly finite derivation of the quantum kink mass. JHEP 1911, 161 (2019). https://doi.org/10.1007/ JHEP11(2019)161. arXiv:1908.06710 [hep-th]

5. R. Rajaraman, Some nonperturbative semiclassical methods in quantum field theory: a pedagogical review. Phys. Rep. 21, 227 (1975). https://doi.org/10.1016/0370-1573(75)90016-2

6. J. Evslin, Well-defined quantum soliton masses without supersymmetry. Phys. Rev. D 101(6), 065005 (2020). https://doi.org/10. 1103/PhysRevD.101.065005. [arXiv:2002.12523 [hep-th]]

7. J. Cotler, M.R. Mohammadi Mozaffar, A. Mollabashi, A. Naseh, Renormalization group circuits for weakly interacting continuum field theories. Fortsch. Phys. 67(10), 1900038 (2019). https://doi. org/10.1002/prop.201900038. arXiv:1806.02831 [hep-th]

8. J.S. Cotler, M. Reza Mohammadi Mozaffar, A. Mollabashi, A. Naseh, Entanglement renormalization for weakly interacting fields. Phys. Rev. D 99(8), 085005 (2019). https://doi.org/10.1103/ PhysRevD.99.085005. [arXiv:1806.02835 [hep-th]]

9. A.M. Jaffe, Divergence of perturbation theory for bosons. Commun. Math. Phys. 1, 127 (1965)

10. J. Glimm, A.M. Jaffe, A lambda phi**4 quantum field theory without cutoffs 1. Phys. Rev. 176, 1945 (1968). https://doi.org/10.1103/ PhysRev.176.1945

11. M. Cohen, The energy spectrum of excitations in liquid helium. Doctoral Thesis at the Caltech, Submitted in November 1955

12. C.M. Bender, T.T. Wu, Anharmonic oscillator. Phys. Rev. 184, 1231 (1969). https://doi.org/10.1103/PhysRev.184.1231

13. S.R. Coleman, Commun. Math. Phys. 31, 259 (1973). https://doi. org/10.1007/BF01646487

14. S.R. Coleman, Subnucl. Ser. 13, 297 (1977)

15. J. Evslin, JHEP 1911, 161 (2019). https://doi.org/10.1007/ JHEP11(2019)161. arXiv:1908.06710 [hep-th]

16. A. Rebhan, P. van Nieuwenhuizen, Nucl. Phys. B 508, 449 (1997). https://doi.org/10.1016/S0550-3213(97)00625-1, https://doi.org/ 10.1016/S0550-3213(97)80021-1 [hep-th/9707163]

17. J.G. Taylor, Solitons as infinite constituent bound states. Ann. Phys. 115, 153 (1978). https://doi.org/10.1016/0003-4916(78)90179-3 